Takashi Wada · Hitoshi Yokoyama • Norihiko Sakai

Yoshiaki Izumiya • Miho Shimizu • Kengo Furuichi

Chikako Segawa - Tsuguho Misaki - Ken-ichi Kobayashi

\title{
Cytokine upregulation in tubulointerstitial nephritis associated with membranous nephropathy
}

Received: May 6, 1998 / Accepted: October 9, 1998

\begin{abstract}
We describe the upregulation of cytokines in a 45 year-old woman with tubulointerstitial nephritis and membranous nephropathy revealed by renal biopsy. She was treated with a combination of prednisolone and cyclosporin. Histological findings showed appreciable improvement, and urinary protein excretion was decreased from $15 \mathrm{~g} /$ day to $1 \mathrm{~g} /$ day. Elevated urinary levels of chemokines, interleukin (IL)-8 and monocyte chemotactic and activating factor (MCAF)/monocyte chemoattractant protein (MCP)1 , and serum levels of tumor necrosis factor (TNF)- $\alpha$ decreased during convalescence; 13 other patients with membranous nephropathy did not show elevation of these cytokines. These results suggest that the upregulation of these cytokines may participate in the pathogenesis of tubulointerstitial nephritis and that combination therapy of prednisolone and cyclosporin may be effective, possibly via inducing a decrease in these cytokines.
\end{abstract}

Key words Tubulointerstitial nephritis - Membranous nephropathy $\cdot$ Cytokine $\cdot$ Chemokine

\section{Introduction}

Tubulointerstitial diseases, including tubulointerstitial nephritis, have a major impact on overall renal function and on the eventual prognosis of renal diseases. ${ }^{1}$ The mechanism by which tubulointerstitial lesions develop remains unclear. Recent studies have revealed that the upregulation of cytokines is involved in the pathogenesis of experimental

T. Wada $(\bowtie) \cdot$ H. Yokoyama $\cdot$ N. Sakai $\cdot$ Y. Izumiya $\cdot$ M. Shimizu K. Furuichi · C. Segawa · T. Misaki · K. Kobayashi

First Department of Internal Medicine, Division of Blood

Purification, Kanazawa University School of Medicine, 13-1 Takaramachi, Kanazawa 920-8641, Japan

Tel. +81-76-265-2000 (ext 3462); Fax +81-76-234-4250

e-mail: twada@med.kanazawa-u.ac.jp tubulointerstitial nephritis models. ${ }^{2,3}$ However, the crucial role of cytokines has not been fully investigated in the pathogenesis of human tubulointerstitial nephritis. Here we describe a patient who developed tubulointerstitial nephritis associated with membranous nephropathy showing the upregulation of cytokines. This suggests that dysregulation of cytokines may be involved in the pathogenesis of human tubulointerstitial nephritis.

\section{Case report}

In May 1990, a 45-year-old woman was admitted to Kanazawa University Hospital for evaluation of insidious edema and proteinuria of several months' duration. She denied any history of drug usage or past history of diseases including urinary abnormalities. Physical examination revealed edema of the face and bilateral lower legs. Urine analysis showed proteinuria of $15.0 \mathrm{~g} /$ day and no microscopic hematuria. Urine sediment showed clustering of leukocytes and some hyaline casts. Urine culture and cytology were negative. Hematological laboratory test results were: hematocrit, $30.7 \%$; red blood count, $3310000 / \mathrm{mm}^{3}$; and hemoglobin, $10.4 \mathrm{~g} / \mathrm{dl}$. Renal function was mildly reduced (24h creatinine clearance (Ccr) $80.6 \mathrm{ml} / \mathrm{min}$ ), while urinary $\beta 2$-microglobulin and N-acetyl-glucosaminidase showed high levels $(20000 \mathrm{ng} / \mathrm{ml}$ and $471.1 \mathrm{IU} / 1$, respectively). Total serum protein was $4.3 \mathrm{~g} / \mathrm{dl}$ (albumin $1.7 \mathrm{~g} / \mathrm{dl}$ ) and serum cholesterol was high $(340 \mathrm{mg} / \mathrm{dl})$. There was marked $\mathrm{IgG}$ hypogammaglobulinemia $(628 \mathrm{mg} / \mathrm{dl})$. Results of serological testing for antinuclear antibody or components of complement were negative or normal. In addition, there were no clinical symptoms suggestive of collagen diseases.

Renal biopsy showed mild diffuse thickening of the glomerular capillary walls accompanied by spike formation. The interstitium was diffusely infiltrated by small round cells, and tubulitis was detected (Fig. 1). Immunofluorescence microscopy revealed granular deposition of immune complex containing IgG and C3 along the glomerular capillary walls. Subepithelial dense deposits were also observed 


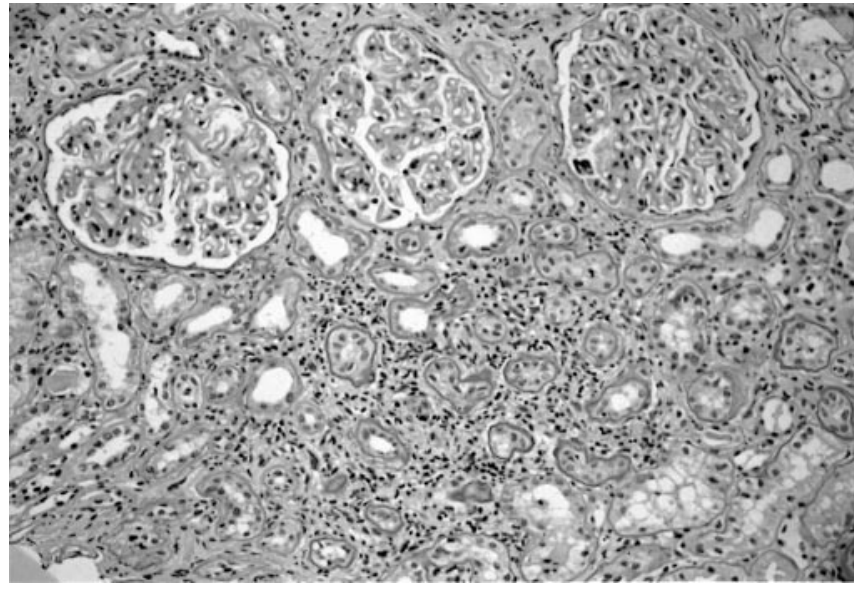

Fig. 1. Renal biopsy revealed tubulointerstitial nephritis associated with membranous nephropathy. Periodic acid Schiff, $\times 320$

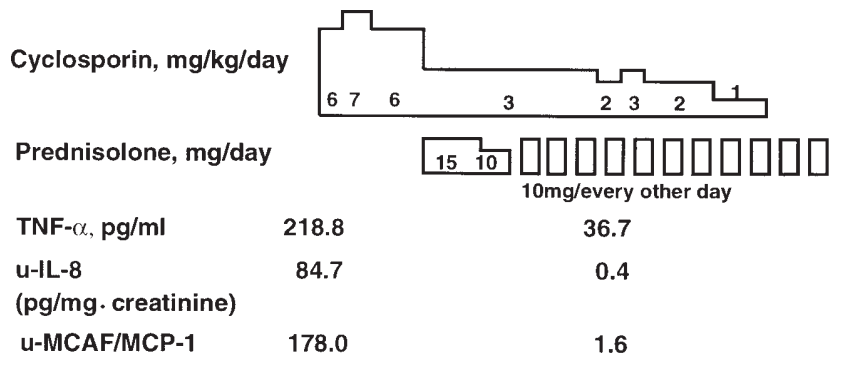

$(\mathrm{pg} / \mathrm{mg} \cdot \mathrm{creatinine}) \quad 178.0 \quad 1.6$

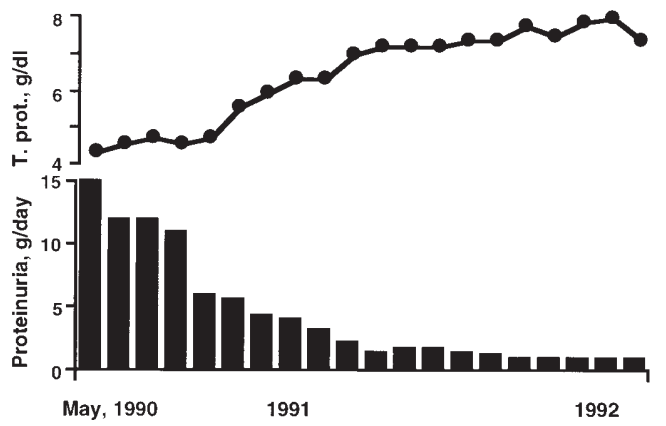

Fig. 2. Clinical course of the patient. $u$-, Urinary; $I L-8$, interleukin-8; $M C A F$, monocyte chemotactic and activating factor; $M C P-1$, monocyte chemoattractant protein-1; TNF- $\alpha$, tumor necrosis factor- $\alpha$

by electron microscopy. Human renal tubular antigen (RTE) was not detected by the indirect immunofluorescence method using specific antihuman RTE rabbit serum (a kind gift from Dr. Naruse ${ }^{4}$ ), as previously described. ${ }^{5}$ Renal biopsies were performed with the informed consent of the patient.

We instituted combined therapy of oral prednisolone (15 mg daily) with cyclosporin (6 mg/kg per day) (Fig. 2). These treatments improved both urinary protein excretion and serum levels of total protein. After the therapy, a rebiopsy specimen of the kidney revealed marked improvement of interstitial cell infiltration and tubulitis. We measured the urinary levels of the chemokines, interleukin8 (IL-8) and monocyte chemotactic and activating factor $(\mathrm{MCAF}) /$ monocyte chemoattractant protein-1 (MCP-1), which are involved in both the recruitment and the activation of neutrophils ${ }^{6}$ and monocytes, respectively, ${ }^{7}$ by enzyme-linked immunosorbent assay (ELISA). Urinary IL8 and MCAF/MCP-1 levels were standardized by the amount of creatinine in the urine. The elevated urinary IL8 level of $84.7 \mathrm{pg} / \mathrm{mg} \cdot$ creatinine (normal range, $<0.2 \mathrm{pg} / \mathrm{mg}$ - creatinine) and the MCAF/MCP-1 level, of $178.0 \mathrm{pg} / \mathrm{mg}$. creatinine (normal range, $<0.4 \mathrm{pg} / \mathrm{mg} \cdot$ creatinine) were found to be dramatically decreased during the patient's immunosuppressive therapy-induced convalescence to $0.4 \mathrm{pg} / \mathrm{mg} \cdot$ creatinine and $1.6 \mathrm{pg} / \mathrm{mg} \cdot$ creatinine, respectively. In contrast, plasma IL-8 was not detected (normal $<16 \mathrm{pg} / \mathrm{ml}$ ) and serum MCAF/MCP-1 was slightly high $(93 \mathrm{pg} / \mathrm{ml}$; normal, $66.1 \pm 15.5 \mathrm{pg} / \mathrm{ml}$, mean \pm SEM). In addition, the elevated serum level of tumor necrosis factor$\alpha(\mathrm{TNF}-\alpha ; 218.8 \mathrm{pg} / \mathrm{ml})$ was decreased to $36.7 \mathrm{pg} / \mathrm{ml}$, detected by ELISA as previously described. ${ }^{8}$

We found that in 13 other patients with membranous nephropathy, urinary levels of IL-8 were below detectable levels, and urinary MCAF/MCP-1 and serum TNF- $\alpha$ levels were not as high $(4.0 \pm 0.1 \mathrm{pg} / \mathrm{mg} \cdot$ creatinine and $2.7 \pm$ $2.5 \mathrm{pg} / \mathrm{ml}$, respectively), as in the patient described. She has received prednisolone $10 \mathrm{mg}$ for maintenance every other day and the proteinuria has never recurred.

\section{Discussion}

We reported here a patient with tubulointerstitial nephritis associated with membranous nephropathy with elevated levels of cytokines. Tubulointerstitial nephritis observed in membranous nephropathy is relatively rare. ${ }^{9}$ This patient denied any history of drug usage or collagen diseases. Katz et al. ${ }^{10}$ reported three patients with anti-tubular basement membrane antibody nephritis associated with membranous nephropathy. Although we did not evaluate anti-tubular basement membrane antibody in this patient, we did not find any deposition of IgG along tubular basement membrane or RTE in glomerular capillaries. Thus, the mechanism of the association of membranous nephropathy and tubulointerstitial nephritis remains to be investigated.

This study revealed elevated levels of urinary IL- 8 and MCAF/MCP-1 in this patient with tubulointerstitial nephritis associated with membranous nephropathy. We did not detect high levels of IL-8 or MCAF/MCP-1 in patients with membranous nephropathy without interstitial nephritis. The most noticeable difference between this patient and the others was in the marked infiltration of mononuclear cells. Although we did not detect the cell sources of the cytokines IL- 8 and MCAF/MCP-1, these data suggest that the upregulation of these chemokines was highly specific for the tubulointerstitial nephritis in this patient. In support of this notion, we have previously reported that MCAF/MCP-1 plays a pivotal role in tubulointerstitial damage and promotes renal dysfunction in human lupus 
nephritis, ${ }^{7}$ IgA nephropathy, ${ }^{11}$ and an experimental glomerulonephritis model. ${ }^{12}$ Furthermore, we previously reported that infiltrating mononuclear cells, tubular epithelial cells, and endothelial cells of the peritubular capillaries were positive for IL- 8 and MCAF/MCP-1 by immunohistochemical and in-situ hybridization analyses. ${ }^{6,7}$ Therefore, even though it is not clear at present whether these cytokines induce tubulointerstitial nephritis or whether tubulointerstitial nephritis lesions release these cytokines, or whether both of these phenomena occur, these results suggest that upregulation of these chemokines may be closely associated with the pathogenesis of tubulointerstitial lesions in this patient.

We found that TNF- $\alpha$ in the serum was also elevated in this patient. TNF- $\alpha$ plays a crucial role in the pathogenesis of interstitial lesions in membranous nephropathy. ${ }^{13}$ Taken together, it appears that once tubular epithelial cells and interstitial infiltrates have been activated by some inflammatory process, IL- $8, \mathrm{MCAF} / \mathrm{MCP}-1$, and TNF- $\alpha$ promote the interstitial inflammatory process through the infiltration and activation of leukocytes.

Urinary and serum levels of these cytokines fell to lower levels during the patient's convalescence induced by glucocorticoid and cyclosporin. The efficacy of cyclosporin in the treatment of membranous nephropathy has been established ${ }^{14}$ as judged by the improvement of the proteinuria. Although the precise mechanism of the effects of this combined therapy on membranous nephropathy and tubulointerstitial nephritis remains to be investigated, one believable mechanism may be the inhibition of cytokines, including IL-8, MCAF/MCP-1, and TNF- $\alpha$. Since glucocorticoids decrease the level of TNF- $\alpha$, the inducers of IL- 8 and MCAF/MCP- $1,{ }^{15}$ via inhibition of the activity of NFKB,${ }^{16}$ glucocorticoids may suppress these cytokines, thereby inhibiting the activation and infiltration of leukocytes. In addition, cyclosporin inhibits T-cell activation via a decrease in IL-2 ${ }^{17}$ which may decrease cytokines produced by T cells directly, or may indirectly decrease these cytokines produced by other inflammatory cells via an inhibitory interaction by inactivated $\mathrm{T}$ cells. Cyclosporin enhances the barrier size-selection of the glomerular basement membrane,$^{14}$ which may lead to the improvement of proteinuria. Collectively, this combined therapy may be effective in membranous nephropathy and tubulointerstitial nephritis, possibly via the improvement of barrier size and via a direct and/or indirect decrease of cytokines, leading to the reduction of immune complex and the inhibition of leukocyte activation and infiltration.

In summary, these results suggest that the upregulation of cytokines may play an important role in the pathogenesis of tubulointerstitial nephritis and that combined therapy with oral prednisolone and cyclosporin is effective in tubulointerstitial nephritis and membranous nephropathy.

Acknowledgments We gratefully acknowledge Prof. Kouji Matsushima (University of Tokyo) for his assistance and advice with the measuring of IL-8 and MCAF/MCP-1 by ELISA. This work was supported by a grant from the Japan Research Foundation for Clinical Pharmacology (TW) and a Grant-in-Aid (No.09671157) from the Ministry of Education, Science, Sport, and Culture of Japan (HY).

\section{References}

1. Kelly CJ, Neilson EG. Tubulointerstitial diseases. In: The Kidney. 5th ed. Philadelphia: WB Saunders, 1996:1655-79.

2. Tang WW, Qi M, Warren JS, Van GY. Chemokine expression in experimental tubulointerstitial nephritis. J Immunol 1997;159:8706.

3. Tang WW, Feng L, Mathison JC, Wilson CB. Cytokine expression, up-regulation of intercellular adhesion molecule-1, and leukocyte infiltration in experimental tubulointerstitial nephritis. Lab Invest 1994;70:631-8.

4. Naruse T, Kitamura K, Miyakawa K, Shibata S. Deposition of renal tubular epithelial antigen along the glomerular capillary walls of patients with membranous glomerulonephritis. J Immunol 1973;110:1163-6.

5. Yokoyama H, Kida H, Asamoto T, Abe T, Koshino Y, Hattori N. Gouty kidney associated with membranous nephropathy: Participation of renal tubular epithelial antigen. Nephron 1986;44:361-4.

6. Wada T, Yokoyama H, Tomosugi N, Hisada Y, Ohta S, Naito T, et al. Detection of urinary interleukin-8 in glomerular diseases. Kidney Int 1994;46:455-60.

7. Wada T, Yokoyama H, Su SB, Mukaida N, Iwano M, Dohi K, et al. Monitoring urinary levels of monocyte chemotactic and activating factor reflects disease activity of lupus nephritis. Kidney Int 1996;49:761-7.

8. Yokoyama H, Takaeda M, Wada T, Ohta S, Hisada Y, Segawa C, et al. Glomerular ICAM-1 expression related to circulating TNF- $\alpha$ in human glomerulonephritis. Nephron 1997;76:425-33.

9. Ivanyi B, Marcussen N, Olsen S. Tubulitis in primary vascular and glomerular renal disease. Pathol Res Pract 1995;191:124557.

10. Katz A, Fish AJ, Santamaria P, Nevins TE, Kim Y, Butkowski RJ. Role of antibodies to tubulointerstitial nephritis antigen in human anti-tubular basement membrane nephritis associated with membranous nephropathy. Am J Med 1992;93:691-8.

11. Yokoyama H, Wada T, Furuichi K, Segawa C, Shimizu M, Kobayashi K, et al. Urinary levels of chemokines (MCAF/MCP-1, IL-8) reflect distinct disease activities and phases of human IgA nephropathy. J Leukocyte Biol 1998;63:493-9.

12. Wada T, Yokoyama H, Furuichi K, Kobayashi K, Harada K, Naruto M, et al. Intervention of crescentic glomerulonephritis in rat by administrating antibodies against monocyte chemotactic and activating factor/monocyte chemoattractant protein-1 (MCAF/ MCP-1). FASEB J 1996;10:1418-25.

13. Honkanen E, von Willebrand E, Teppo AM, Tornroth T, Gronhagen-Riska C. Adhesion molecules and urinary tumor necrosis factor- $\alpha$ in idiopathic membranous glomerulonephritis. Kidney Int 1998;53:909-17.

14. Ambalavanan S, Fanvel JP, Sibley RK, Myers BD. Mechanism of the anti-proteinuric effect of cyclosporin in membranous nephropathy. J Am Soc Nephrol 1996;7:290-8.

15. Schwarz M, Radeke H, Resch K, Uciechowski P. Lymphocytederived cytokines induce sequential expression of monocyte- and $\mathrm{T}$ cell-specific chemokines in human mesangial cells. Kidney Int 1997;52:1521-31.

16. Adock IM, Brown CR, Gelder CM, Shirasaki H, Peters MJ, Barnes PJ, Luster AD. Effects of glucocorticoids on transcription factor activation in human peripheral mononuclear cells. Am J Physiol 1995;268:C331-8.

17. Emmel EA, Verweij CL, Durand DB, Higgins KM, Lacy E, Grabtree GR. Cyclosporin A specifically inhibits function of nuclear proteins involved in $\mathrm{T}$ cell activation. Science 1989;246:1617-20. 